[13] G. R. Tristram. In The Proteins, New-York, vol. 1, part A, 216. 1953.

[14] S. J. Rowland et M. Zein-el-Dine. J. Dairy Sci., 10, 266. 1939.

[15] C. Vanderzant et W. R. Tennison. Abst. paper 55th ann. Meet. Am. Dairy Sci. Assoc., 1960, in J. Dairy Sci., 43, 854. 1960.

[16] K. Shiga, R. Shimizu et H. Hама. Bull. Nat. Inst. Agric. Sci. (Japan), série G, no $18,1.1959$.

[17] J. M. Treece, L. O. Gilmore et N. S. Fischmeister. J. Dairy Sci., 727, 41. 1958.

[18] J. M. Treece, L. O. Gilmore et N. S. Fischmeister. J. Dairy Sci., 42, 367. 1959.

\title{
COMMENT CONCEVOIR UNE SURVEILLANCE DES POLLUTIONS RADIOACTIVES DU LAIT
}

\author{
par \\ G. MICHON \\ Service d'Hygiène atomique \\ et de Radiopathologie \\ C. E. A.
}

Le lait, aliment essentiel, fait l'objet d'une surveillance et de contrôles vigilants régis par de nombreux textes législatifs qui protègent ses qualités marchandes et sanitaires.

Le développement de l'utilisation de l'énergie nucléaire pose et posera de nouveaux problèmes d'hygiène et le lait doit bénéficier de contrôles portant sur sa pollution radioactive éventuelle.

Les animaux herbivores sont particulièrement exposés aux contaminations radioactives et les laits qu'ils produisent, du fait de leurs compositions salines et du métabolisme particulier de la glande mammaire, n'échappent point aux pollutions. De ce fait, le lait est un intégrateur de contamination et son analyse permet de retrouver des radioéléments que l'on ne décèle qu'avec beaucoup de difficultés sur un échantillon végétal de taille moyenne.

\section{I. - ORgANISATION DES PRÉLÈVEMENTS}

Le but de la surveillance du lait consiste à déterminer quelle est la contribution de cet aliment à la contamination de l'homme. Cette participation est importante pour les radioéléments de la famille des alcalino-terreux, car le lait reste tout au long de notre vie, dans les pays européens, le vecteur essentiel du calcium alimentaire. 
Les prélèvements doivent être effectués de manière à couvrir une part très importante de la production laitière. Ils doivent permettre une extrapolation peu hasardeuse à l'ensemble de la consommation, sans pourtant aboutir à un nombre prohibitif d'analyses qui restent à l'heure actuelle difficiles, longues et coûteuses.

\section{Lieux des prélèvements}

Deux méthodes de prélèvements peuvent être envisagées, l'une consistant à effectuer le contrôle au stade de la production, l'autre au stade de la consommation.

L'avantage essentiel d'un contrôle effectué au stade de la production réside dans le fait que le prélèvement n'est pas séparé de son contexte agronomique. Il correspond à une région bien déterminée et permet d'utiles comparaisons et recoupements avec les données des mesures de radioactivité effectuées sur l'air et sur l'eau.

Dans ce cas, il y a intérêt à éliminer les faibles variations qui peuvent apparaître dans la région retenue en effectuant les prélèvements sur un lait de grand mélange provenant d'un périmètre dee collecte bien défini. Les grandes laiteries coopératives, par exemple, satisfont bien à ces exigences.

Par contre, lorsqu'il s'agit d'étudier l'ingestion de radioéléments par les individus d'une population donnée, celle d'une grande ville, par exemple, ou celle d'une région exposée aux pollutions, mais non productrice de lait, le lieu de prédilection du prélèvement se situe au niveau du circuit de distribution. Pour réduire cependant le nombre des analyses, il convient de réaliser un échantillon composite en mélangeant des volumes aliquotes des quantités de lait distribuées par les différentes firmes qui assurent le ravitaillement de la population considérée.

\section{Fréquence des prélèvements}

La fréquence des prélèvements dépend du type de surveillance qu'il convient d'effectuer. Il est possible de définir trois types de surveillance qui peuvent par ailleurs, correspondre à des zones géographiques différentes.

Tout d'abord, une surveillance générale, destinée à obtenir une idée moyenne de ce qui se passe sur le territoire national. Cette surveillance peut bénéficier des prélèvements effectués au stade de la production et de la consommation. La fréquence des analyses peut alors n'être que mensuelle. 
Certaines régions peuvent, par suite de l'implantation de sites nucléaires ou de toute autre installation industrielle utilisant des radioéléments ou en produisant, paraître plus exposées à des risques de contamination. Dans de telles parties du territoire, la surveillance doit être plus vigilante en resserrant les points de prélèvement et en effectuant des analyses plus fréquentes, hebdomadaires par exemple.

Enfin, il n'est pas possible d'éliminer complètement le cas d'un incident voire d'un accident survenant à une de ces installations et entraînant une pollution importante du milieu ambiant. Dans ce cas, les analyses deviennent plus aisées ; elles peuvent et doivent être effectuées pratiquement sans délai et dans tout le périmètre contaminé, les prélèvements seront effectués suivant un quadrillage serré et avec une fréquence accrue, journalière ou même correspondant aux deux traites quotidiennes.

Les circonstances peuvent aussi modifier dans une certaine mesure la fréquence des prélèvements, sinon des analyses. Dans les périodes de fortes fluctuations des retombées radioactives, une interprétation correcte ne peut être obtenue que si l'on élimine les variations quotidiennes et si l'on obtient une valeur intégrée sur la période considérée. Ce résultat peut être obtenu aisément au stade de la production, en effectuant un petit prélèvement quotidien aliquote de la quantité de lait traitée par la firme et en réunissant ces prélèvements en un seul échantillon, destiné à l'analyse mensuelle par exemple.

Dans le cas où le taux de retombée reste pratiquement constant, de même que dans les régions éloignées des sources de pollution, le prélèvement au lieu d'être quotidien peut devenir bimensuel ou mensuel. Cette fréquence peut être retenue lorsque le contrôle s'effectue au stade de la distribution du produit.

Cependant si d'un échantillon au suivant, l'analyse fait apparaître de fortes variations, il convient de revenir à l'échantillonnage journalier ; ce dernier, dans ce cas, est fort pesant.

\section{Nature du prélèvement et quantité à prélever}

Les prélèvements peuvent porter indifféremment, dans la plupart des cas, sur du lait en nature, du lait concentré ou en poudre. Le lait en poudre présente l'avantage d'être facile à expédier, de bien se prêter aux analyses de spectrométrie et d'être facile à minéraliser. De plus, dans les usines de transformation, il existe un personnel technique qui peut effectuer, dans de bonnes condi- 
tions, les prélèvements quotidiens et assurer l'homogénéisation de l'échantillon final, ce qui offre des garanties supplémentaires de représentativité de ce dernier.

Dans le cas d'une surveillance effectuée à la production, le lait en poudre est l'échantillon de choix. Si les prélèvements sont effectués sur les circuits de distribution, ou en cas d'incident, ils porteront sur le lait liquide qui reste la forme d'utilisation la plus usuelle.

En l'absence de données contraires, l'on peut considérer que les techniques utilisées en fromagerie n'entraînent pas de discrimination entre le calcium et le strontium et que, par conséquent, le rapport du strontium radioactif au calcium reste le même dans le lait et le produit de transformation.

Cette donnée n'est peut être pas extrapolable à d'autres éléments chimiques et, dans certains cas, il sera utile de déterminer l'appauvrissement ou l'enrichissement qui apparaît dans l'une des fractions au cours de la fabrication du produit dérivé.

Les quantités à prélever dépendent de la teneur et de la nature des radioéléments à rechercher et à doser. Faible, de l'ordre du litre, lorsqu'il s'agira d'un incident, cette quantité sera beaucoup plus importante dans les zones non menacées et en l'absence de fortes retombées radioactives. La quantité prélevée, dès qu'une opération chimique est indispensable, doit permettre d'effectuer l'analyse en double. Il ne semble pas que le volume puisse alors être inférieur à dix litres.

\section{II. - TRAITEMENT ET MESURE DES ÉCHANTILLONS}

\section{Traitement}

Le traitement que doit subir l'échantillon dépend des mesures que l'on désire exécuter.

D'une manière générale, toutes les opérations de transformation de l'échantillon pour le mettre sous une forme mieux adaptée à la mesure envisagée, s'effectueront à une température inférieure à la température de volatilisation des combinaisons chimiques de l'élément recherché présentes dans le lait.

Les recherches de substances émettrices de rayonnement $\gamma$ peuvent être entreprises sur le lait liquide ou en poudre ; par contre, pour le dosage d'émetteurs de rayonnement $\beta$, il est indispensable de minéraliser l'échantillon et ensuite, dans bien des cas, d'extraire et purifier le radioélément. 


\section{Mesure d'une radioactivité $\gamma$}

La mesure d'une activité $\gamma$ s'effectue de préférence avec un cristal scintillateur sensible au rayonnement $\gamma$ et un photomultiplicateur.

La mesure de l'activité $\gamma$ globale consiste à recueillir toutes les impulsions délivrées par le photomultiplicateur dans un ensemble électronique de dénombrement.

Pour être utile, un tel ensemble doit être stable dans le temps. Afin de pouvoir comparer des résultats obtenus dans différents laboratoires, il est nécessaire d'obtenir des renseignements précis sur ces installations. Outre la nature et la taille du détecteur, les dimensions de la protection, la géométrie retenue pour les mesures, il est indispensable de définir avec précision, la gamme d'énergie dans laquelle opère l'installation.

Il est plus intéressant de disposer d'un sélecteur qui permet de classer les impulsions en fonction de leur énergie, de tracer un spectre de l'activité $\gamma$ de l'échantillon et de déceler ainsi la présence de tel ou tel radionuclide émetteur $\gamma$.

La détermination exacte de la teneur de l'échantillon en un radionuclide émetteur $\gamma$ est complexe, elle nécessite un étalonnage précis de l'installation pour ce nuclide en présence des autres radionuclides qui polluent habituellement le lait.

Il est utile de tirer avantage de la présence du potassium naturel en l'utilisant comme étalon interne émetteur $\gamma$. En effet, la teneur en potassium peut être déterminée aisément par analyse spectrographique de flamme et la radioactivité due au ${ }^{40} \mathrm{~K}$ calculées.

La comparaison des résultats obtenus par différents laboratoires ne pourra s'effectuer dans des conditions satisfaisantes qu'au moyen d'étalons provenant d'un même stock et distribués à tous.

\section{Mesure d'une radioactivité $\beta$}

Jusqu'à nouvel ordre, la meilleure manière d'étudier le rayonnement $\beta$ consiste à utiliser un compteur Geiger-Muller.

La comparaison des résultats sera possible si l'on définit l'épaisseur de la fenêtre du compteur et si l'on détermine le rendement global de l'installation pour un échantillon d'un sel de potassium, réalisé dans des conditions normalisées de masse et d'épaisseur. Ces conditions resteront les mêmes pour les échantillons de cendre de lait.

La détermination de la radioactivité $\beta$ globale des cendres n'a pas de sens physique par elle-même, cependant la succession des mesures peut, en l'absence de contaminations nouvelles, donner 
une idée de la stabilité de la pollution et éviter d'entreprendre systématiquement une détermination difficile comme celle du strontium.

Dans le même esprit, mais dans le but d'obtenir un renseignement plus fin, on peut rechercher la radioactivité $\beta$ du précipité d'oxalate obtenu sur des cendres de lait. En éliminant le potassium, on élimine les fluctuations saisonnières ou autres qui lui sont propres et l'on saisit mieux la constance ou les variations des pollutions artificielles.

Il n'en demeure pas moins impératif d'effectuer des déterminations précises des différents isotopes susceptibles d'être rencontrés sur au moins 20 p. 100 des échantillons, avec au minimum deux déterminations par an pour chacun de ces éléments en chaque point de prélèvement.

La détermination précise de la teneur en un radionuclide émetteur $\beta$ nécessite la concentration et l'extraction, puis la purification de cet élément. Il convient alors de déterminer de façon très précise, le rendement des installations de mesure pour le spectre d'électrons émis par le corps.

Cette détermination devra se faire pour tous les laboratoires à partir d'échantillons provenant de la même solution étalon.

La détermination du rendement complet des opérations chimiques et physiques, devra se faire à partir d'échantillons de poudre de lait ou de cendre de lait provenant d'un stock commun et dont la teneur pour l'élément considéré aura été déterminée par plusieurs laboratoires spécialisés.

En procédant de la sorte, il n'est pas indispensable d'imposer à tous les laboratoires des techniques chimiques ou physiques uniformes qui risquent d'être périmées dès la mise en place du dispositif de contrôle. Chaque laboratoire devra néanmoins fournir le détail des techniques qu'il utilise.

\section{III. - RADIOÉLÉMENTS PARTIGULIERS \\ A RECHERGHER ET A DOSER}

Le choix de ces radioéléments est conditionné :

10 d'une part, par la physiologie même de la glande mammaire et la composition saline du lait;

$2^{\circ}$ d'autre part, par les risques de pollution générale ou propre à une région donnée.

Dans le cadre des pollutions générales du globe, il est essentiel, actuellement, d'obtenir des données précises sur la teneur du lait en : 
- éléments alcalins et notamment ${ }^{137} \mathrm{Cs}$;

- éléments alcalino-terreux et notamment ${ }^{90} \mathrm{Sr}$.

$\mathrm{Au}$ voisinage des sites nucléaires ou en cas d'accident, outre les éléments déjà signalés et dont l'étude est impérative, il conviendra de déceler la présence éventuelle d'iode 131.

D'autres éléments pourront, en fonction des risques particuliers dus à la présence d'un site nucléaire ou d'une usine être recherchés. La liste ne peut en être déterminée a priori, chaque cas devant être étudié séparément.

La pollution du lait peut s'effectuer hors de la voie physiologique, notamment au cours de la traite ou par l'utilisation d'eau polluée au rinçage des pots de collecte ou des installations de transformation. Il ne faut donc pas écarter la possibilité d'une contamination par des radioéléments qui franchissent difficilement la barrière intestinale et la barrière mammaire, mais qui, néanmoins, peuvent ainsi se retrouver de façon régulière dans le lait commercialisé.

\section{IV. - EXPRESSION DES RÉSULTATS}

L'unité d'expression de la radioactivité est le curie dont les sousmultiples sont le millicurie $\left(10^{-3}\right)$, le microcurie $\left(10^{-6}\right)$, le nanocurie $\left(10^{-9}\right)$ et le picocurie $\left(10^{-12}\right)$.

La radioactivité sera toujours rapportée à l'unité de volume, c'est-à-dire au litre.

Les mesures de radioactivité globale peuvent être exprimées comme s'il s'agissait uniquement de potassium naturel, en admettant que le spectre électronique de l'échantillon est identique à celui du potassium. On obtiendra ainsi des " picocuries équivalents » de ${ }^{40} \mathrm{~K}$.

De même la radioactivité d'un précipité d'oxalate peut être attribuée à un ensemble de radioéléments et ne peut done, de ce fait, être exprimée en sous multiples du curie. Il est possible néanmoins d'admettre que toute cette radioactivité est due à la présence du plus dangereux, c'est-à-dire au ${ }^{90} \mathrm{Sr}$ et l'exprimer en " picocuries équivalents » de ${ }^{90} \mathrm{Sr}$.

On obtient ainsi une estimation pessimiste de la teneur en ${ }^{90} \mathrm{Sr}$ qui peut éviter, en cas de contrôle urgent, d'aller plus avant dans l'analyse.

En ce qui concerne le strontium et le césium, outre l'activité spécifique rapportée au litre, il y aura intérêt à exprimer aussi le strontium en picocuries rapportés au gramme de calcium présent dans l'échantillon, pour tenir compte de similitudes physiologiques 
et le césium en picocuries rapportés au gramme de potassium présent dans l'échantillon du fait que celui-ci peut servir d'étalon interne pour les déterminations spectrométriques $\gamma$.

Les autres éléments éventuellement recherchés et dosés, seront exprimés en picocuries par litre.

Lorsque la recherche du $\mathrm{Sr}$ ou du $\mathrm{Ca}$, du fait de teneurs trop faibles, ne permettra pas de conclure avec certitude, il conviendra d'indiquer qu'elle est inférieure à la quantité la plus petite qui peut être mesurée avec certitude au moyen des installations dont dispose le laboratoire.

Une telle surveillance est actuellement techniquement possible. Cependant elle ne peut se concevoir sans la coopération de tous ceux qui se préoccupent de questions laitières. Deux types de laboratoires doivent être envisagés : les premiers relativement nombreux, effectuent les analyses les plus élémentaires, les seconds, mieux équipés, peuvent pousser les analyses aux limites actuelles de détection et de mesure. L'implantation de ce premier type de laboratoire pourrait se concevoir dans des laboratoires déjà existants qui recevraient l'appareillage électronique adéquat. Quant aux laboratoires du second type, qui nécessitent des investissements importants, il serait préférable qu'ils n'existent qu'à de rares exemplaires.

L'ensemble des résultats doit être centralisé pour donner lieu à une exploitation rationnelle. Il sera alors possible d'avoir des données précises sur les pollutions éventuelles du lait et d'assurer le contrôle efficace dont le lait mérite bien d'être le premier aliment à être doté.

\section{LE LAIT : FORMULES NOUVELLES}

$$
\text { par }
$$

\section{G. THIEULIN}

Sécurité bactériologique, maintien des valeurs biologiques, caractères organoleptiques favorables, constituent, sans nul doute, la meilleure qualification du lait.

Dans ce triple but, nous connaissons parfaitement les règles à respecter pour établir les conditions favorables de production d'un

(1) Bull. Acad. Méd., 1961, 145, 25. 